\title{
Structure determination of Murine Norovirus NS6 proteases with C-terminal extensions designed to probe protease- substrate interactions
}

Humberto Fernandes, Eoin N Leen, Hamlet Cromwell Jnr, Marc-Philipp Pfeil, Stephen Curry

Noroviruses are positive-sense single-stranded RNA viruses. They encode an NS6 protease that cleaves a viral polyprotein at specific sites to produce mature viral proteins. In an earlier study we obtained crystals of murine norovirus (MNV) NS6 protease in which crystal contacts were mediated by specific insertion of the C-terminus of one protein (which contains residues P5-P1 of the NS6-7 cleavage junction) into the peptide binding site of an adjacent molecule, forming an adventitious protease-product complex. We sought to reproduce this crystal form to investigate protease-substrate complexes by extending the C-terminus of NS6 construct to include residues on the C-terminal $\left(P^{\prime}\right)$ side of the cleavage junction. We report the crystallization and crystal structure determination of inactive mutants of murine norovirus NS6 protease with C-terminal extensions of one, two and four residues from the N-terminus of the adjacent NS7 protein (NS6 1', NS6 2', NS6 4'). We also determined the structure of a chimeric extended NS6 protease in which the P4 P4' sequence of the NS6-7 cleavage site was replaced with the corresponding sequence from the NS2-3 cleavage junction (NS6 4'2|3).

The constructs NS6 $1^{\prime}$ and NS6 2' yielded crystals that diffracted anisotropically. We found that, although the uncorrected data could be phased by molecular replacement, refinement of the structures stalled unless the data were ellipsoidally truncated and corrected with anisotropic B-factors. These corrections significantly improved phasing by molecular replacement and subsequent refinement.

The refined structures of all four extended NS6 proteases are very similar in structure to the mature MNV NS6 - and in one case reveal additional details of a surface loop.

Although the packing arrangement observed showed some similarities to those observed in the adventitious protease-product crystals reported previously, in no case were specific protease-substrate interactions observed. 
2 Structure determination of Murine Norovirus NS6 proteases with C-terminal extensions

3 designed to probe protease-substrate interactions

6 Humberto Fernandes*

7 Department of Life Sciences, Sir Ernst Chain Building, Imperial College London, SW7 2AZ, UK.

8 Present address: Laboratory of Genomic Engineering, Institute of Biochemistry and Biophysics, Polish

9 Academy of Sciences, Pawinskiego 5A, 02-106 Warsaw, Poland

11 Eoin N. Leen

12 Department of Life Sciences, Sir Ernst Chain Building, Imperial College London, SW7 2AZ, UK.

14 Hamlet Cromwell Jr

15 Department of Life Sciences, Sir Ernst Chain Building, Imperial College London, SW7 2AZ, UK.

Marc-Philipp Pfeil

18 Department of Life Sciences, Sir Ernst Chain Building, Imperial College London, SW7 2AZ, UK.

19 Present address: Department of Biochemistry, University of Oxford, South Parks Road, Oxford OX1

20 3QU, UK

Stephen Curry

*Corresponding author.

34 Tel/Fax: +48 225921310

35 E-mail: hfernandes@ibb.waw.pl

Key words: Anisotropic diffraction, Data processing, Elliptical truncation, $R$ factors, Refinement, Crystal contacts, Crystal structure;

Running title: Murine norovirus NS6 - anisotropic diffraction and crystal structures 
39 Noroviruses are responsible for over half of the outbreaks of gastroenteritis worldwide (Karst, 2010).

40 They belong to the Caliciviridae, a family of positive-sense, single-stranded RNA viruses with a

$41 \sim 7.5 \mathrm{~kb}$ genome that generally contains three open reading frames (ORF) (Jiang et al., 1993; Lambden

42 et al., 1993; Glass et al., 2000); a novel fourth ORF was recently identified in Murine norovirus (MNV)

43 (McFadden et al., 2011). Whereas ORF2, ORF3 and ORF4 each encode single proteins, ORF1 codes

44 for a large polyprotein $(\sim 190 \mathrm{kDa})$ that is processed by the virally-encoded protease at five specific

45 sites to release the six 'mature' non-structural proteins (NS1/2-NS7) - and an array of functional

46 precursors - that are required for virus replication (Belliot et al., 2003; Sosnovtsev et al., 2006;

47 Muhaxhiri et al., 2013). The viral NS6 protease present within the C-terminal half of the polyprotein

48 performs all the processing, including its own autocatalytic release from the precursor (Liu, Clarke \&

49 Lambden, 1996; Belliot et al., 2003; Sosnovtsev et al., 2006; Scheffler et al., 2007).

50 Crystal structures have been determined for the NS6 proteases from several norovirus strains (Chiba

51 virus, Murine norovirus, Norwalk virus, Southampton norovirus) (Nakamura et al., 2005; Zeitler, Estes

52 \& Prasad, 2006; Hussey et al., 2011; Kim et al., 2012; Leen, Baeza \& Curry, 2012; Muhaxhiri et al.,

53 2013). The solution structure and dynamics of Norwalk virus NS6 have also been analysed by NMR

54 spectroscopy (Takahashi et al., 2013). Norovirus NS6 is a cysteine protease with a chymotrypsin-like

55 fold: two $\beta$-barrel domains separated by a cleft that contains a Cys-His-Asp/Glu catalytic triad similar

56 in arrangement to the Ser-His-Asp triad characteristic of serine proteases (Allaire et al., 1994;

57 Matthews et al., 1994). Calicivirus NS6 is related in sequence and structure to the picornavirus 3C

58 proteases, which have the same role in polyprotein processing for these single-stranded RNA viruses

59 (Leen, Baeza \& Curry, 2012). 
60 Previously our lab determined the structure of full-length Murine norovirus NS6 (residues 1-183)

61 (Leen, Baeza \& Curry, 2012). Strikingly, adventitious crystal contacts placed the C-terminus of one

62 molecule in the active site of another, thereby generating the structure of a protease-product complex

63 that plausibly represents the final step of the trans cleavage by NS6 at the NS6-NS7 junction. An

64 almost identical packing arrangement was obtained in a different space-group with crystals of Norwalk

65 virus NS6 protease (Muhaxhiri et al., 2013). These structures, together with the structures of di-, tri-

66 and penta-peptidyl substrate analogues bound to human norovirus NS6 structures (Hussey et al., 2011;

67 Kim et al., 2012; Muhaxhiri et al., 2013) revealed details of the specific contacts made by the P5-P1

68 residues of peptidyl products of the protease (Fig. 1); in particular they showed the anchoring of P1-

69 Gln by specific H-bond interactions with Thr 134 and His 157 (Fig. 1a), and the accommodation of the

70 hydrophobic side-chains of P2-Phe and P4-Leu in apolar pockets (Fig. 1b). However, to date there are

71 no structural data on protease-substrate complexes that might uncover details of the interactions made

72 by the protease with the C-terminal, prime side of noroviral peptide cleavage junctions (residues P1'-

$\left.73 \mathrm{P} 4^{\prime}\right)$.

74 We reasoned that catalytically inactive MNV NS6 constructs extended at the C-terminus might be able

75 to crystallise with the same packing contacts as we had observed for the full-length protein, and that

76 this could therefore give us a convenient way to investigate the structures of NS6-substrate complexes.

77 To this end we extended an inactive MNV NS6 construct (which incorporates a C139A mutation to

78 knock out the active site nucleophile) by adding residues from NS7, which is immediately downstream

79 in the polyprotein. We made three constructs, NS6 1', NS6 2' and NS6 4', which were extended by 1,2

80 and 4 residues respectively, generating proteases that contain substrates that correspond to P4-P1', $\mathrm{P} 4-$

81 P2' and P4-P4' of the NS6-NS7 cleavage junction. To investigate the structural variation in substrate 
82 recognition we also made an NS6 chimera which interchanged the residues P4-P4' of the NS6-7

83 cleavage junction with the sequence of the NS2-3 junction from MNV (NS6 4' 2|3).

84 Proteins expressed from all four constructs were crystallised and their structures determined at high

85 resolution by X-ray crystallography. The diffraction patterns from crystals of NS6 1' and NS6 2'

86 exhibited marked anisotropy, which stalled the crystallographic refinement at high R-factors. However,

87 this was overcome by the successful application of an anisotropic data correction procedure (Sawaya,

88 2014).

89 The modified MNV NS6 constructs all crystallised with packing arrangements that were distinct from

90 that observed in crystals we previously obtained with the mature NS6 protease - the final 183-residue

91 protein released after processing of the viral polyprotein (Leen, Baeza \& Curry, 2012; Muhaxhiri et al.,

92 2013). Unfortunately, in no case did the packing arrangements involve insertion of the cleavage

93 junction in the extended C-terminus into the active site of an adjacent protein in a way that allowed the

94 formation of specific protease-substrate interactions. The four new structures reported here therefore do

95 not provide any new information on the mode of binding of the P1'-P4' residues of junctions cleaved

96 by MNV NS6. However they do confirm the MNV NS6 protease structure and, in at least one case,

97 reveal the structure for the loop connecting $\beta$-strands cII and dII that was disordered in the previously

98 reported structure (Leen, Baeza \& Curry, 2012).

99

100 Materials and methods

101 Cloning and purification of MNV NS6 variants. C-terminally extended constructs of the murine

102 norovirus NS6 protease (UniProt accession no. Q80J95; residues 995-1177), inactivated by mutation of 
103 the active site Cys 139 to $\mathrm{Ala}(\mathrm{C} 139 \mathrm{~A})$ were generated by the polymerase chain reaction using the 104 inactive full length MNV NS6 as a template (Leen, Baeza \& Curry, 2012). The same forward primer 105 was used throughout (5'- CATCATGGATCCGCCCCAGTCTCCATCTGG). To create constructs 106 extended by one (Gly; NS6 1'), two (Gly-Pro; NS6 2') or four residues (Gly-Pro-Pro-Met; NS6 4')

107 beyond the natural C-terminus of NS6, we used the reverse primers, 5'-

108 ATGATGAAGCTTAGCCCTGGAACTCCAGAGCCTCAA, 5'-

109 CATCATAAGCTTACGGGCCCTGGAACTCCAGAGCCTCAAGTGTGGGTTCTCCGTGAGT and 110 5'-CATCATAAGCTTACATCGGCGGGCCCTGGAACTCCAGAGCCTCAAGTG respectively. To

111 generate the chimera with the P4-P4' cleavage site of NS2-3 (NS6 4' 2|3) the reverse primer 5"-

112 TACTACAAGCTTAATCAAACGGGCCTTCCGCCTGCCAAGCCTCAAGTGTGGGTTCTCCGTG

113 AGT was used. PCR products were digested with BamHI and HindIII and ligated into the pETM-11

114 vector as described previously for full-length NS6 (Leen, Baeza \& Curry, 2012). The expressed MNV

115 NS6 variants thus contain a thrombin-cleavable N-terminal His ${ }_{6}$ tag; processing by thrombin leaves a

116 Gly-Ser di-peptide preceding the Ala1 residue at the N-terminus of our constructs. All plasmid insert

117 sequences were confirmed by DNA sequencing (Eurofins MWG Operon). NS6 1' and NS6 2'

118 constructs were transformed into E. coli BL21-CodonPlus (Stratagene) and grown in lysogeny broth

119 (LB) supplemented with $25 \mu \mathrm{g} / \mathrm{mL}$ kanamycin and $35 \mu \mathrm{g} / \mathrm{mL}$ chloramphenicol, while NS6 4' and

120 NS6 4' 2|3 constructs were transformed into E. coli BL21(DE3) pLysS (Promega) and grown in LB

121 supplemented with $25 \mu \mathrm{g} / \mathrm{mL}$ kanmycin and $35 \mu \mathrm{g} / \mathrm{mL}$ chloramphenicol. For large-scale protein

122 expression, $1 \mathrm{~L}$ of LB was inoculated with overnight cultures. The cultures were incubated at $37^{\circ} \mathrm{C}$

123 with shaking at 220 RPM and protein expression was induced for 3 to 4 hours by addition of $1 \mathrm{mM}$

124 isopropyl $\beta$-D-1-thiogalactopyranoside (IPTG) once the cultures had attained an OD at $600 \mathrm{~nm}$ of 1.0.

125 Cells were harvested by centrifugation at $4000 \mathrm{~g}$ for $10 \mathrm{~min}$, and pellets frozen at $-80^{\circ} \mathrm{C}$. 
126 E. coli pellets containing over-expressed NS6 1' and NS6 2' were re-suspended in lysis buffer (50 mM

127 Tris $\mathrm{pH}$ 8.0, $300 \mathrm{mM} \mathrm{NaCl}, 1 \mathrm{mM}$ dithiothreitol (DTT)) supplemented with $0.5 \mathrm{mM}$ PMSF, $2 \mathrm{mg} / \mathrm{ml}$

128 lysozyme, and $0.1 \%(\mathrm{v} / \mathrm{v})$ Triton X-100 and the cells disrupted by sonication. Cell debris was removed

129 by centrifugation of the bacteria lysate at $29000 \mathrm{~g}$ for $1 \mathrm{~h}$. Clarified lysates were incubated with

$1301 \mathrm{mg} / \mathrm{mL}$ protamine sulphate for $20 \mathrm{~min}$ at $4^{\circ} \mathrm{C}$ to precipitate nucleic acid contaminants, which were

131 removed by re-centrifugation at $29000 \mathrm{~g}$ for $20 \mathrm{~min}$. The lysate was loaded onto TALON metal affinity

132 resin (Clontech) in a gravity flow column. After washing with 25 volumes of lysis buffer, 25 volumes

133 of lysis buffer containing $5 \mathrm{mM}$ imidazole, and finally a further 25 volumes of lysis buffer containing

$13410 \mathrm{mM}$ imidazole, the MNV NS6 proteins were eluted with a step gradient to $100 \mathrm{mM}$ imidazole. The

$135 \mathrm{His}_{6}$ tag was removed during overnight dialysis in lysis buffer in the presence $2 \mathrm{mM} \mathrm{CaCl}_{2}$, and 10

136 units of thrombin (Sigma-Aldrich) per mg of MNV NS6 protein. Following dialysis, uncleaved

137 material and cleaved tags were removed in a second round of TALON purification. As a final polishing

138 step, the unbound fractions containing cleaved MNV NS6 variants were pooled, concentrated and

139 loaded onto a Superdex-75 size-exclusion column (mounted on AKTA FPLC, GE Healthcare)

140 equilibrated with $50 \mathrm{mM}$ Tris $\mathrm{pH}$ 8.0, $100 \mathrm{mM} \mathrm{NaCl}, 1 \mathrm{mM}$ DTT (SEC buffer).

141 In the case of NS6 4' and NS6 4' $2 \mid 3$ constructs the above protocol (and additives) were used but the

142 lysis buffer was composed instead of $50 \mathrm{mM}$ HEPES $\mathrm{pH}$ 6.5, $300 \mathrm{mM} \mathrm{NaCl}, 1 \mathrm{mM}$ DTT. Size-

143 exclusion chromatography was performed in $25 \mathrm{mM}$ Tris $\mathrm{pH}$ 8.0, $200 \mathrm{mM} \mathrm{NaCl}, 5 \mathrm{mM}$ DTT.

145 Crystallisation of MNV NS6 variants. All crystallisation experiments were performed with a protein 146 concentrations of $10-14.5 \mathrm{mg} / \mathrm{mL}$ in the SEC buffer used for the final purification step. Efforts to use 147 the original crystallisation conditions to reproduce the MNV NS6 protein crystals with the packing 
148 arrangement that would place the C-terminus of one molecule in the active site of a neighbour (Leen,

149 Baeza \& Curry, 2012) failed for the four extended constructs generated in this study. Seeding

150 equilibrated drops with fragments from extant NS6 crystals generated very small crystals that appeared

151 similar in habit to the original crystals of MNV NS6 but they did not diffract appreciably.

152 We therefore widened the search for crystallisation conditions, carrying out screening on a sub-

153 microlitre scale by sitting-drop vapour-diffusion. $100 \mathrm{~nL}$ protein solution at $14 \mathrm{mg} / \mathrm{mL}$ was mixed with

$154100 \mathrm{~nL}$ reservoir solution and equilibrated against $90 \mu \mathrm{L}$ of reservoir solution using a Mosquito

155 automated pipetting system (TTP LabTech) and the following commercial crystallisation screens:

156 Wizard $1 \& 2$ (Emerald Biosystems), PACT Premier, JCSG+, Morpheus and PGAScreen (Molecular

157 dimensions). Larger crystals were grown from initial hits by sitting-drop vapour diffusion at $18^{\circ} \mathrm{C}$ in

158 CompactClover Plates EBS-XPT (Jena Bioscience).

159 Useable crystals of MNV NS6 1' were obtained by mixing $1 \mu \mathrm{L}$ of protein with $1 \mu \mathrm{L}$ mother liquor

160 consisting of 10\% (v/v) poly-ethylene glycol (PEG) 10000, 20\% (v/v) ethylene glycol, $0.1 \mathrm{M}$

161 MES/Imidazole pH 6.3. Crystals with cuboid shape appeared between 2 and 5 days and grew to full

162 size $\left(80 \times 50 \times 50 \mu_{\mathrm{m}}\right)$ in about 15 days and were flash-cooled in liquid nitrogen prior to data collection

163 without any additional cryo-protectant.

164 Crystals of MNV NS6 2' were obtained in 15\% (v/v) PEG 3350, 0.1 M glycine, $0.1 \mathrm{M}$ Na-citrate pH

165 7.0. They grew as long rods in 1-2 days and achieved full size $(400 \times 30 \times 30 \mu \mathrm{m})$ in 5-10 days. The

166 crystals were cryo-protected in mother liquor supplemented with 15\% (v/v) PEG 200 and flash-cooled 167 in liquid nitrogen. 
168 Crystals of NS6 4' and NS6 4' 2|3 grew from 0.2 M KSCN, 0.1 M Bis-Tris propane pH 6.5-7.5, 20\%

169 w/v PEG 3350. They were cryo-protected adjusting the mother liquor solution to a final concentration 170 of $30 \%(\mathrm{v} / \mathrm{v})$ PEG 3350.

171 X-Ray data collection and processing. X-Ray diffraction data from crystals of NS6 $1^{\prime}$ and NS6 2'

172 were collected on a Pilatus 6M-F detector at the I03 beamline at the Diamond Light Source (Didcot, 173 UK). For the MNV NS6 1' crystals a $2.3 \AA$ data set of 200 frames was collected with an oscillation 174 width of $1^{\circ}$ per frame. Diffraction images were integrated and scaled using the CCP4 program suite 175 (Winn et al., 2011). Data-collection statistics are summarized in Table 1.

176 For the MNV NS6 $2^{\prime}$ crystals, 720 frames with a $0.5^{\circ}$ oscillation were collected. A $3.1 \AA$ dataset was 177 integrated and scaled as described above; see Table 1 for data collection statistics.

178 MNV NS6 4' and NS6 4' 2|3 crystals data was collected in-house using a Rigaku MicroMax-007 HF-M

179 X-ray generator and a Saturn 944+ CCD detector. Data sets of 316 and $3440.5^{\circ}$ oscillation frames

180 were collected for NS6 4' and NS6 4' $2 \mid 3$ crystals at 2.47 and $2.42 \AA$ respectively. The data were

181 processed and scaled as described above.

182 All four datasets were submitted to the UCLA MBI Diffraction Anisotropic Server

183 (http://services.mbi.ucla.edu/anisoscale) for anisotropic analysis. Following the server indication of 184 severe anisotropy diffraction of the MNV NS6 1' and NS6 2' crystals both data sets were 185 truncated/scaled using the server default values, in particular using a 3.0 cut-off for F/sigma (Strong et 186 al., 2006; Sawaya, 2014).

187 Phasing, model building and refinement. Molecular-replacement phasing was performed in Phaser 188 (McCoy et al., 2007) using the crystal structure of full-length MNV NS6 (PDB entry 4ASH) (Leen, 
189 Baeza \& Curry, 2012) pruned of double conformations and the initial 6 and terminal 11 residues to

190 avoid biasing the conformations of the termini.

191 The MNV NS6 1' and NS6 2' structures obtained from molecular replacement were subjected to

192 restrained refinement using REFMAC (Murshudov et al., 2011), in the CCP4 program suite

193 (Collaborative Computational Project, 1994). Molecular replacement solutions of NS6 4' and NS6 4'

$1942 \mid 3$ were refined using Phenix refine (Adams et al., 2010). All manual model adjustments were made in

195 Coot (Emsley \& Cowtan, 2004).

196

197

Results and discussion

198

\section{Structure determination}

199 Datasets collected for MNV NS6 1' and NS6 2' were initially scaled and truncated - at $2.3 \AA$ and 3.1

$200 \AA$ A respectively - with the assumption in each case that the diffraction was isotropic even though some 201 anisotropy was evident in the diffraction images. This approach resulted in data sets with relatively 202 high values of $R_{\text {merge }}$ and low signal-to-noise ratios $[I / \sigma(I)]$ for the highest-resolution shells of data 203 (Table 1). The MNV NS6 1' crystals were determined to be composed of four molecules in the 204 asymmetric unit of the $C 2$ unit cell, which is consistent with a solvent content of 50.3\% (Matthews, 205 1968). MNV NS6 $2^{\prime}$ crystals belong to space-group $P 6_{1} 22$ and were determined to contain two 206 molecules in the asymmetric unit, with slightly higher solvent content of 55.3\%.

207 Initial structural factors (processed isotropically) were used for molecular replacement, and in the case 208 of MNV NS6 1' produced four possible solutions with a highest LLG of 2723 and TFZ of 19.7.

209 However, initial refinement of the model obtained by molecular replacement stalled at relatively high 
210 values of $R_{\text {work }}(\sim 33 \%)$ and $R_{\text {free }}(\sim 39 \%)$. A similar problem was encountered with initial attempts to 211 refine the MNV NS6 2' model.

212 At this stage we re-visited the processed data to determine if the anisotropy was at the root of the 213 refinement problems. Plots of F/sigma against resolution for each of the 3 principal axes revealed the 214 severity of the anisotropy of the diffraction from both crystals (Fig. 2a, b); when truncated at F/sigma < 2153.0 along each axis, the spread of data was ellipsoidal in appearance. The server identified the $c^{*}$ axis 216 as stronger diffracting than the $a^{*}$ and $b^{*}$ directions in each case, and detected anisotropic $\Delta B$ values of $21768.6 \AA^{2}$ and $51.0 \AA^{2}$ for MNV NS6 1' and NS6 2', respectively. Anisotropic $\Delta B$ reports the 218 directionality dependence of the intensity falloff with resolution

219 (http://services.mbi.ucla.edu/anisoscale/). Following the F/sigma analysis, the MNV NS6 1' data were 220 truncated to $3.1 \AA, 2.3 \AA$, and $2.1 \AA$ along $a^{*}, b^{*}$ and $c^{*}$, respectively. To create a nominally isotropic 221 data set, $B$-factor corrections of $39.2,-9.8$ and $-29.4 \AA^{2}$ were applied to the observed structure factors 222 along the $a^{*}, b^{*}$ and $c^{*}$ directions respectively. The reduction in the anisotropy of the corrected data in 223 the $\mathrm{a}^{*} \mathrm{c}^{*}(\mathrm{hol})$ plane can been seen by comparing Fig. $2 \mathrm{c}$ and $2 \mathrm{~d}$.

224 Following anisotropy analysis the MNV NS6 2' data was truncated to $3.2 \AA, 3.2 \AA$, and $2.7 \AA$ along $a^{*}$, $225 b^{*}$ and $c^{*}$ directions. To generate the nominally isotropic data set, $B$-factor correction of 17.0, 17.0 and $226-34.0 \AA^{2}$ along the same axes were applied to the observed structure factors. The anisotropic truncation 227 of the data, with the new limit of $2.7 \AA$ along the $c^{*}$ axis, resulted in a dramatic increase in the number 228 of unique reflections from 8555 in the "pre-treated" data to 12786.

MNV NS6 4' or NS6 4' 2|3 data sets plots of F/sigma against resolution for each of the 3 principal axes 230 revealed only mild anisotropy of the diffraction from both crystals (anisotropic $\Delta B$ values of $13.3 \AA^{2}$ 
231 and $15.4 \AA^{2}$ for MNV NS6 4' and NS6 4' 2|3, respectively). In these cases no directional-dependent 232 truncation of the data was applied.

233 After taking account of the anisotropy of the data, the axes-dependent truncated and corrected structure 234 factors were then used to repeat the molecular replacement phasing of the NS6 1' data and resulted in 235 significantly better solutions. The best solution had an LLG of 3051 and TFZ of 32.3. Moreover, the 236 model showed immediate improvement in the early cycles of refinement $\left(R_{\text {work }} \sim 31 \% ; R_{\text {free }} \sim 36 \%\right)$ and 237 yielded electron density maps that were much more interpretable (Fig. 2e, f). Multiple cycles of 238 refinement and model building lead to a structure characterized by $R_{\text {work }}$ of $23.0 \%$ and $R_{\text {free }}$ of $27.8 \%$.

239 Anisotropic treatment of the MNV NS6 2' data also improved the molecular replacement outcome, 240 with a unique solution found by Phaser in contrast with the two possible solutions of the uncorrected 241 data. The LLG value increased markedly from 385 to 887 while there was a slight drop in the value of 242 TFZ (from 19.2 to 18.8). Nevertheless, this resulted in immediate improvements in refinement: $R_{\text {work }}$ dropped from 32.5 to $30.3 \%$ while $R_{\text {free }}$ was reduced from 42.0 to $39.0 \%$. These statistics were further improved to $R_{\text {work }}$ of $25.4 \%$ and $R_{\text {free }}$ of $30.3 \%$ in the final structure.

245 The translation Z-scores and LLG's for the Phaser molecular replacement solutions for MNV NS6 4' 246 are 13.8 and 1042, respectively, and for MNV NS6 4' 2|3 27.1 and 1394, respectively. The crystals structures of NS6 4' and NS6 4' $2 \mid 3$ were refined to $R$ factors $\left(R_{\text {work }} / R_{\text {free }}\right)$ of 20.9/25.2 and 21.0/26.2 \%, 248 respectively. 
251 Overall, the structure of the protease core domains for NS6 1', NS6 2', NS6 4' and NS6 2|3 4' is very 252 similar to that reported previously for MNV NS6 (Leen, Baeza \& Curry, 2012) - (root mean squared 253 differences are less than $1 \AA$ ) (Fig. 3). For three of the four new structures (NS6 2', NS6 4' and 254 NS6 2|3 4') it was possible to identify conserved crystal contacts, involving a two-fold symmetric 255 packing arrangement largely mediated by the short $\mathrm{N}$-terminal helix and the loop connecting $\beta$-strand 256 aII and bII (Fig. 3b). In each case the C-terminus of at least one protein molecule in the asymmetric 257 unit was observed to extend away from the body of the protease and to interact with a neighbouring 258 molecule in the crystal — as had been observed previously for MNV NS6. Moreover, the extended C259 terminus invariably inserted into the peptide-binding groove formed between loops bII-cII and eII-fII 260 loops (Fig. 4). Disappointingly however, in no case did the extended peptide make interactions that 261 were consistent with specific contacts with the protease active site. We will briefly describe each of the 262 four new structures before turning to the question of why in no case a protease-substrate complex was 263 obtained.

264 MNV NS6 1' crystallised in space group $C 2$ with four molecules in the asymmetric unit. This structure 265 is notable for the fact that, in contrast to the other three structures reported here and the structures 266 previously reported for Norwalk virus NS6 protease (Zeitler, Estes \& Prasad, 2006; Muhaxhiri et al., 267 2013), the electron density map was of sufficient quality in all four molecules of the asymmetric unit to 268 permit the incorporation of the loop between the $\beta$-strands cII and dII (Fig. 3a). Superposition of the 269 four copies of NS6 1' indicates some structural variation in this loop — the $\mathrm{C}_{\alpha}$ positions vary by $1-2 \AA$ 270 - consistent with the notion that it is rather flexible (Fig. 3a). 
271 In three of the molecules in the asymmetric unit the C-terminus is disordered beyond residue 173 272 (chains A and D) or residue 174 (chain C). The electron density for the last four residues modelled in 273 chain D (Ala 170 to Gly 173) is poor, presumably due to disorder. Nevertheless, we included these 274 residues in the final model because removal increased the $R$-factor.

275 Although the full C-termini of chains A, C and D of the NS6 1' could not be modelled, the electron 276 density map revealed additional features that could be built as short stretches of polypeptide. These 277 correspond to portions of the missing C-termini but, because of discontinuities in the electron density, 278 it is not possible to unambiguously ascribe which monomer they belong to. These short segments were 279 present in the asymmetric unit, and were modelled as (i) a stretch of five residues (Chain E) that could 280 be assigned confidently as corresponding to residues Leu 180 to Gly 184 that lies near to the active site 281 of a neighbouring chain A; and (ii) an extended portion of electron density that was not of sufficient 282 quality to identify the amino acid side chains and was built as a seven-residue poly-Ala peptide (chain 283 F), that lies between chains A and D and may be part of a symmetry-related molecule of chain A. 284 However, in neither case were specific interactions with the putative active sites of neighbour 285 monomers observed.

286 In contrast to chains $\mathrm{A}, \mathrm{C}$ and $\mathrm{D}$, the electron density for the $\mathrm{B}$ chain in the asymmetric unit was 287 288 labelled Gly 184) in the refined model (Fig. 4b). The C-terminus of Chain B of NS6 1' beyond Gly 173 289 extends to make contacts with chain B from an adjacent asymmetric unit to which it is related by a 290 two-fold symmetry axis. This two-fold symmetric packing arrangement is reminiscent of the inter291 protein contacts previously observed for the full length NS6 (Leen, Baeza \& Curry, 2012) (Fig. 4a).

292 However, although the C-terminus of NS6 1' comes close to the substrate-binding site of neighbouring 
293 proteases in the crystal, it does not reach far enough and makes none of the specific contacts needed to 294 position the scissile bond (Gln 183-Gly 184) in the active site.

295 MNV NS6 $2^{\prime}$ crystallised in space group $P 6_{1} 22$ with two molecules in the asymmetric unit. The 296 electron density indicates the positions of residues 3-181 in chain A and 4-181 in chain B, but is not 297 good enough to allow complete modelling of the N- or C-termini. The two monomers found in the 298 asymmetric unit are related by a quasi-2-fold symmetry axis and form an apparent homodimer with an 299 interface area of $1097 \AA^{2}$ (Fig. 4c). In addition to neighbourly contacts made by the two C-termini, 300 dimerization within the crystal is stabilised by hydrogen bonds involving Ser 51, Ser 111 and Val 113 301 of chain A and Ser 51, Ser 52, Ser 111 and Val 113 of chain B. Although the C-termini within the 302 asymmetric unit each embrace the other monomer, reaching into the groove formed between the bII-cII 303 and eII-fII loops, they are again not located within the substrate-binding site.

304 MNV NS6 4' crystallised in space group $C 2$ but in a unit cell that is very different from the $C 2$ crystals 305 obtained with MNV NS6 1' (Table 1) and that has only one molecule in the asymmetric unit. The 306 electron density was sufficient to build a model that starts at residue 4 and ends at residue 179. 307 Although the extended C-terminus of NS6 once again reaches between loops bII-cII and eII-fII of its 308 near neighbour, the relation between the two molecules does not involve two-fold or quasi two-fold 309 symmetry (Fig. 4d). However, no specific contacts are made by the C-terminal peptide with the 310 substrate binding site.

311 NS6 4' $2 \mid 3$ crystallised with two molecules in a $P 1$ unit cell. The modelled monomers start at residue 4 312 and end at residue 182; in contrast to the other structures reported here, the A chain lack density for the 313 tip of the eII-fII loop so residues 162-163 were omitted. The A and B chains each extend their C314 termini into the cleft formed between the bII-cII and eII-fII loops of a neighbouring molecule (Fig. 4e, 
315 f). The packing arrangements are distinct but rather similar and, once again, lack specific contacts that 316 are consistent with productive substrate binding.

\section{Implications of the structures}

319 With the extended NS6 protease constructs designed for this study we aimed to exploit our earlier

320 finding that the mature protease crystallised with the C-terminus of one molecule inserted into peptide

321 binding site of a neighbouring protein in a way that revealed specific protease-product interactions

322 (Leen, Baeza \& Curry, 2012). We had hoped to obtain the same packing interaction with the C-

323 terminally extended complexes in order to determine the structures or protease-substrate complexes

324 that would reveal the details of the interactions made by the amino acids in positions $\mathrm{P} 1^{\prime}-\mathrm{P} 4{ }^{\prime}$ of the 325 substrate, but in each of the four cases that we probed, extension of the C-terminus resulted in novel 326 packing arrangements, none of which captured a proper protease-substrate reaction.

327 Why did we not get the same crystal form as for the full-length protein? At present it is difficult to give 328 a definitive answer to this question. Although we screened extensively for crystallisation conditions,

329 we cannot claim to have searched exhaustively and it may be that further efforts might yet succeed.

330 Moreover, it is well known that even very modest changes to protein constructs may alter substantially

331 the way that they crystallise, though it is worth noting that a similar strategy to explore the specific 332 interactions of different protease-product complexes was applied successfully to Norwalk virus NS6

333 (Muhaxhiri et al., 2013). In preliminary investigations using nuclear magnetic resonance (NMR) to

334 investigate solution structures of extended MNV NS6 protease structures, we have obtained evidence 335 to suggest that their C-termini may have a propensity to fold into the active site of the molecule that it 336 belongs to, making a cis interaction (Fernandes, Pastore \& Curry, unpublished data). This may explain 
337 why trans interactions were not observed in our crystals but in turn raises a further question: why was 338 this conformational state not captured in the crystal form? One possibility is that the concentration of 339 proteins inevitably involved in crystallisation somehow destabilizes the cis interaction but does not 340 necessarily capture a catalytically competent trans interaction between the cleavage junction within the 341 extended C-terminus and a protease neighbour. Why this would be the case, particularly when a 342 protease-substrate complex would be expected to be a relatively stable state, remains a mystery.

\section{Acknowledgements}

345 We thank staff at the Diamond Light Source (Didcot, UK) for assistance with data collection. 


\section{References}

348

349

Adams PD, Afonine PV, Bunkoczi G, Chen VB, Davis IW, Echols N, Headd JJ, Hung LW, Kapral GJ, GrosseKunstleve RW, McCoy AJ, Moriarty NW, Oeffner R, Read RJ, Richardson DC, Richardson JS, Terwilliger TC, and Zwart PH. 2010. PHENIX: a comprehensive Python-based system for macromolecular structure solution. Acta Crystallogr. D Biol. Crystallogr. 66:213-221.

Allaire M, Chernaia MM, Malcolm BA, and James MN. 1994. Picornaviral 3C cysteine proteinases have a fold similar to chymotrypsin-like serine proteinases. Nature 369:72-76.

Belliot G, Sosnovtsev SV, Mitra T, Hammer C, Garfield M, and Green KY. 2003. In vitro proteolytic processing of the MD145 norovirus ORF1 nonstructural polyprotein yields stable precursors and products similar to those detected in calicivirus-infected cells. J. Virol. 77:10957-10974.

Collaborative Computational Project N. 1994. The CCP4 suite: programs for protein crystallography. Acta Crystallogr. D Biol. Crystallogr. 50:760-763.

Emsley P, and Cowtan K. 2004. Coot: model-building tools for molecular graphics. Acta Crystallogr. D Biol. Crystallogr. 60:2126-2132.

Glass PJ, White LJ, Ball JM, Leparc-Goffart I, Hardy ME, and Estes MK. 2000. Norwalk virus open reading frame 3 encodes a minor structural protein. J. Virol. 74:6581-6591.

Hussey RJ, Coates L, Gill RS, Erskine PT, Coker SF, Mitchell E, Cooper JB, Wood S, Broadbridge R, Clarke IN, Lambden PR, and Shoolingin-Jordan PM. 2011. A structural study of norovirus 3C protease specificity: binding of a designed active site-directed peptide inhibitor. Biochemistry 50:240-249.

Jiang X, Wang M, Wang K, and Estes MK. 1993. Sequence and genomic organization of Norwalk virus. Virology 195:51-61.

Karst SM. 2010. Pathogenesis of noroviruses, emerging RNA viruses. Viruses 2:748-781.

Kim Y, Lovell S, Tiew KC, Mandadapu SR, Alliston KR, Battaile KP, Groutas WC, and Chang KO. 2012. Broad-spectrum antivirals against $3 \mathrm{C}$ or $3 \mathrm{C}$-like proteases of picornaviruses, noroviruses, and coronaviruses. J. Virol. 86:11754-11762. 
Lambden PR, Caul EO, Ashley CR, and Clarke IN. 1993. Sequence and genome organization of a human small round-structured (Norwalk-like) virus. Science 259:516-519.

Leen EN, Baeza G, and Curry S. 2012. Structure of a murine norovirus NS6 protease-product complex revealed by adventitious crystallisation. PloS ONE 7:e38723.

Liu B, Clarke IN, and Lambden PR. 1996. Polyprotein processing in Southampton virus: identification of 3Clike protease cleavage sites by in vitro mutagenesis. J. Virol. 70:2605-2610.

Matthews BW. 1968. Solvent content of protein crystals. J. Mol. Biol. 33:491-497.

Matthews DA, Smith WW, Ferre RA, Condon B, Budahazi G, Sisson W, Villafranca JE, Janson CA, McElroy HE, Gribskov CL, and et al. 1994. Structure of human rhinovirus 3C protease reveals a trypsin-like polypeptide fold, RNA-binding site, and means for cleaving precursor polyprotein. Cell 77:761-771.

McCoy AJ, Grosse-Kunstleve RW, Adams PD, Winn MD, Storoni LC, and Read RJ. 2007. Phaser crystallographic software. J. Appl. Crystallogr. 40:658-674.

McFadden N, Bailey D, Carrara G, Benson A, Chaudhry Y, Shortland A, Heeney J, Yarovinsky F, Simmonds P, Macdonald A, and Goodfellow I. 2011. Norovirus regulation of the innate immune response and apoptosis occurs via the product of the alternative open reading frame 4. PLoS Pathogens 7:e1002413.

Muhaxhiri Z, Deng L, Shanker S, Sankaran B, Estes MK, Palzkill T, Song Y, and Prasad BV. 2013. Structural basis of substrate specificity and protease inhibition in Norwalk virus. J. Virol. 87:4281-4292.

Murshudov GN, Skubak P, Lebedev AA, Pannu NS, Steiner RA, Nicholls RA, Winn MD, Long F, and Vagin AA. 2011. REFMAC5 for the refinement of macromolecular crystal structures. Acta Crystallogr. D Biol. Crystallogr. 67:355-367.

Nakamura K, Someya Y, Kumasaka T, Ueno G, Yamamoto M, Sato T, Takeda N, Miyamura T, and Tanaka N. 2005. A norovirus protease structure provides insights into active and substrate binding site integrity. $J$. Virol. 79:13685-13693.

Sawaya MR. 2014. Methods to refine macromolecular structures in cases of severe diffraction anisotropy. Methods Mol Biol 1091:205-214. 
397 Scheffler U, Rudolph W, Gebhardt J, and Rohayem J. 2007. Differential cleavage of the norovirus polyprotein 398 precursor by two active forms of the viral protease. J. Gen. Virol. 88:2013-2018.

399 Schrodinger, LLC. 2010. The PyMOL Molecular Graphics System, Version 1.3r1.

400 Sosnovtsev SV, Belliot G, Chang KO, Prikhodko VG, Thackray LB, Wobus CE, Karst SM, Virgin HW, and

401 Green KY. 2006. Cleavage map and proteolytic processing of the murine norovirus nonstructural 402 polyprotein in infected cells. J. Virol. 80:7816-7831.

403 Strong M, Sawaya MR, Wang S, Phillips M, Cascio D, and Eisenberg D. 2006. Toward the structural genomics 404 of complexes: crystal structure of a PE/PPE protein complex from Mycobacterium tuberculosis. Proc. Natl. $405 \quad$ Acad. Sci. USA 103:8060-8065.

406 Takahashi D, Hiromasa Y, Kim Y, Anbanandam A, Yao X, Chang KO, and Prakash O. 2013. Structural and 407 dynamics characterization of norovirus protease. Protein Sci. 22:347-357.

408 Winn MD, Ballard CC, Cowtan KD, Dodson EJ, Emsley P, Evans PR, Keegan RM, Krissinel EB, Leslie AG, 409 McCoy A, McNicholas SJ, Murshudov GN, Pannu NS, Potterton EA, Powell HR, Read RJ, Vagin A, and 410 Wilson KS. 2011. Overview of the CCP4 suite and current developments. Acta Crystallogr. D Biol. 411 Crystallogr. 67:235-242.

412 Zeitler CE, Estes MK, and Prasad BV. 2006. X-ray crystallographic structure of the Norwalk virus protease at 413 1.5-A resolution. J. Virol. 80:5050-5058. 


\section{1}

Figure 1. Specific protease-product interactions observed in the original crystals of MNV NS6 ${ }^{\text {pro. }}$.

(a) The $\mathrm{N}$ - and C-terminal domains of one protease molecule are coloured green and orange respectively. The C-terminus of an adjacent protease that is accommodated specifically in the substrate-binding site (residues P1-P5) is shown as a stick model with grey carbon atoms. Hydrogen-bonds are indicated as dashed lines. (b) Same view as in panel a but with the protease surface shown to illustrate the binding pockets involved in substrate recognition. This figure is a modified version of Fig. 3 from (Leen, Baeza \& Curry, 2012) which was published under a Creative Commons CC-BY licence. All structural figures were made with PyMOL (Schrodinger, 2010).
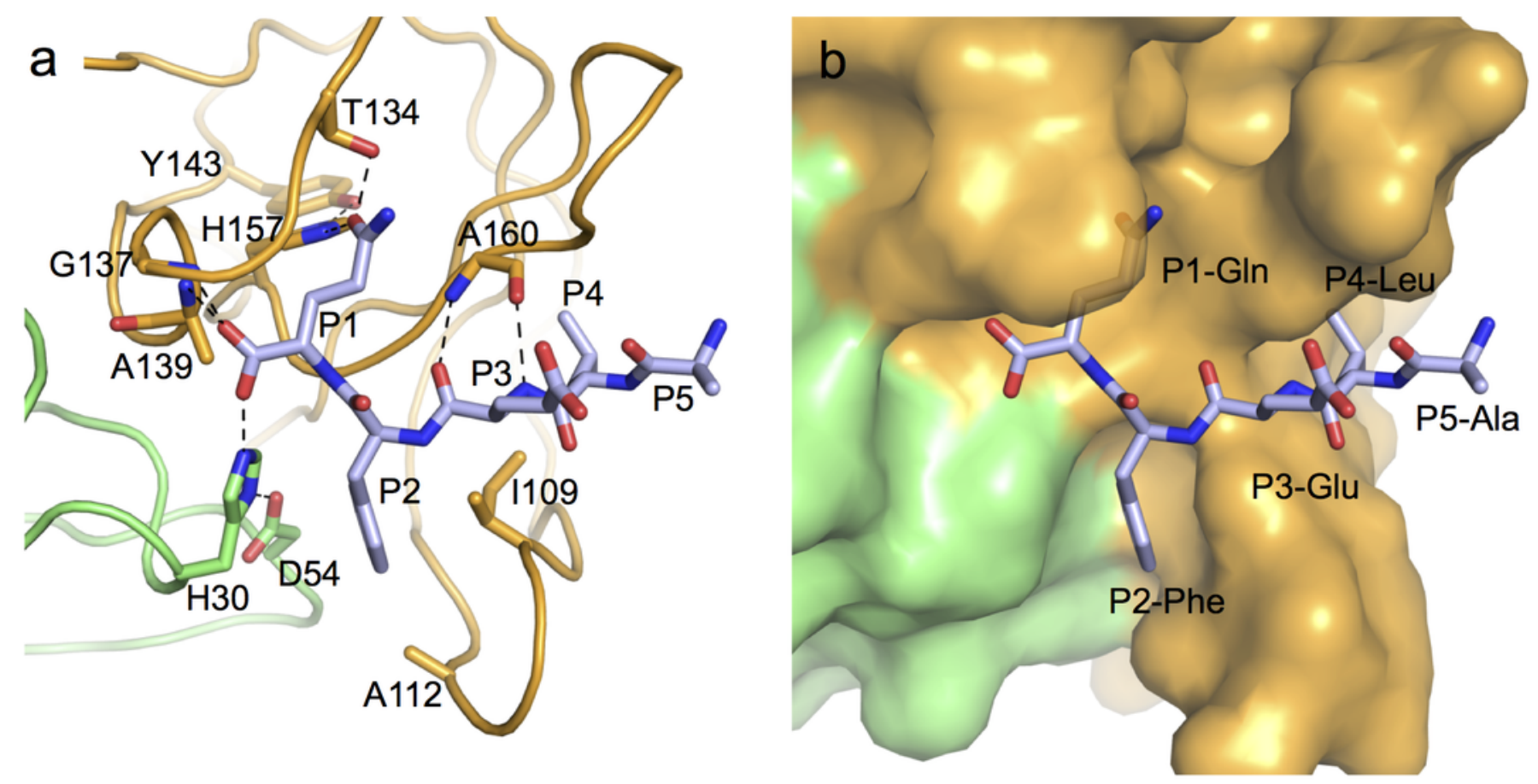


\section{Table $\mathbf{1}$ (on next page)}

Table 1. Data-collection and model refinement statistics. 


\begin{tabular}{|c|c|c|c|c|}
\hline \multicolumn{5}{|l|}{ Data Collection } \\
\hline & MNV NS6 1' & MNV NS6 $2^{\prime}$ & MNV NS6 4' & MNV NS6 4' $2 \mid 3$ \\
\hline Radiation source & Diamond I03 & Diamond I03 & $\begin{array}{l}\text { Rigaku MicroMax- } \\
007 \text { HF-M }\end{array}$ & $\begin{array}{l}\text { Rigaku MicroMax- } \\
007 \text { HF-M }\end{array}$ \\
\hline Wavelength $(\AA)$ & 1.000 & 1.000 & 1.54 & 1.54 \\
\hline Detector & Pilatus 6M-F & Pilatus $6 \mathrm{M}-\mathrm{F}$ & Saturn $944+$ CCD & Saturn $944+$ CCD \\
\left.\hline${\operatorname{Resolution~} \operatorname{limits}^{1}(\AA)}^{(}\right)$ & $\begin{array}{l}70.93-2.3 \\
(2.42-2.3)\end{array}$ & $\begin{array}{l}68.02-3.1 \\
(3.31-3.1)\end{array}$ & $\begin{array}{l}38.11-2.472 \\
(2.561-2.472)\end{array}$ & $\begin{array}{l}19.27-2.417 \\
(2.503-2.417)\end{array}$ \\
\hline Space group & $C 2$ & $P 6_{1} 22$ & $C 2$ & $P 1$ \\
\hline Unit-cell parameters $\left(\AA,^{\circ}\right)$ & $\begin{array}{l}a=99.64 \\
b=111.86 \\
c=81.29 \\
\beta=119.24\end{array}$ & $\begin{array}{l}a=136.04 \\
c=82.39\end{array}$ & $\begin{array}{l}a=88.19 \\
b=35.36 \\
c=52.81 \\
\beta=105.98\end{array}$ & $\begin{array}{l}a=35.52 \\
b=47.32 \\
c=53.07 \\
\alpha=104.45 \\
\beta=91.53 \\
\gamma=110.61\end{array}$ \\
\hline Mosaicity $\left({ }^{\circ}\right)$ & 0.42 & 0.70 & 0.67 & 1.11 \\
\hline No. of unique reflection & 34593 & 8555 & 5711 & 10817 \\
\hline Multiplicity & $3.5(3.6)$ & $5.7(6.0)$ & $3.0(2.3)$ & $1.8(1.8)$ \\
\hline$\langle I / \sigma(I)\rangle^{2}$ & $6.7(1.2)$ & $10.9(2.1)$ & $19.63(6.94)$ & $6.7(2.31)$ \\
\hline Completeness (\%) & $99.7(99.8)$ & $99.8(99.6)$ & $99.03(92.44)$ & $91.14(66.47)$ \\
\hline$R_{\text {merge }}(\%)^{3}$ & $8.4(8.6)$ & $8.5(91.5)$ & $3.4(10.6)$ & $9.3(27.1)$ \\
\hline Overall $B$ factor $\left(\AA^{2}\right)$ & 54.4 & 94.3 & 27.1 & 24.9 \\
\hline \multicolumn{5}{|l|}{ Model Refinement } \\
\hline $\begin{array}{l}\text { No. of Non-hydrogen } \\
\text { atoms/waters }\end{array}$ & $5356 / 93$ & $2563 / 0$ & $1218 / 22$ & $2562 / 107$ \\
\hline$R_{\text {work }}(\%)^{4}$ & 23.0 & 25.4 & $20.9(27.2)$ & $21.0(28.3)$ \\
\hline$R_{\text {free }}(\%)^{5}$ & 27.8 & 30.3 & $25.2(37.1)$ & $26.2(36.8)$ \\
\hline RMSD bonds $(\AA ̊)^{6}$ & 0.012 & 0.012 & 0.009 & 0.003 \\
\hline RMSD bond angles $\left({ }^{\circ}\right)$ & 1.605 & 1.610 & 1.08 & 0.67 \\
\hline $\begin{array}{l}\text { Ramachandran plot } \\
\text { (\% favoured/allowed) }\end{array}$ & $89.3 / 10.7$ & $88.8 / 11.2$ & $97.5 / 2.5$ & $96.1 / 3.9$ \\
\hline PDB Identifier & $4 \times 2 v$ & $4 \times 2 w$ & $4 \times 2 x$ & $4 \times 2 y$ \\
\hline
\end{tabular}


$4 \quad{ }^{1}$ Values in parentheses refer to the highest resolution shell of data.

$5{ }^{2}\langle I / \sigma(I)\rangle$ is the mean signal-to-noise ratio, where $I$ is the integrated intensity of a measured reflection

6 and $\sigma(I)$ is the estimated error in the measurement.

$7 \quad{ }^{3} R_{\text {merge }}=100 \times \Sigma_{\mathrm{hkl}} \mid \mathrm{I}_{\mathrm{j}}(\mathrm{hkl})-\left\langle\mathrm{I}_{\mathrm{j}}(\mathrm{hkl})\right\rangle / \Sigma_{\mathrm{hkl}} \Sigma_{\mathrm{j}} \mathrm{l}(\mathrm{hkl})$, where $\mathrm{I}_{\mathrm{j}}(\mathrm{hkl})$ and $\left\langle\mathrm{I}_{\mathrm{j}}(\mathrm{hkl})\right\rangle$ are the intensity of

8 measurement $\mathrm{j}$ and the mean intensity for the reflection with indices hkl, respectively.

$9 \quad{ }^{4} R_{\text {work }}=100 \times \Sigma_{\mathrm{hk} \mid}|| \mathrm{F}_{\mathrm{obs}}|-| \mathrm{F}_{\text {calc }}\left|/ \Sigma_{\mathrm{hk}}\right| \mathrm{F}_{\text {obs }} \mid$.

$10{ }^{5} R_{\text {free }}$ is the $R_{\text {work }}$ calculated using a randomly selected $5 \%$ sample of reflection data that were omitted

11 from the refinement.

$12{ }^{6} R M S D$, root-mean-squared deviations (from ideality). 
Figure 2. Analysis and correction of the anisotropic diffraction observed for crystals of NS6 $1^{\prime}$ and NS6 2'.

F/sigma vs. Bragg spacings for each of the cell directions for (a) NS6 1' and (b) NS6 2' respectively. Pseudo-precession images of the anisotropy in the $a^{*} c^{*}(h 0 /)$ plane for NS6 1 ' (c) before and (d) after correction. $2 \mathrm{~F}_{\mathrm{o}}-\mathrm{F}_{\mathrm{c}}$ electron density maps contoured at $2 \sigma$ after one round of refinement of the molecular replacement solutions obtained with Phaser (McCoy et al., 2007) for NS6 1' (e) before and (f) after anisotropic correction.
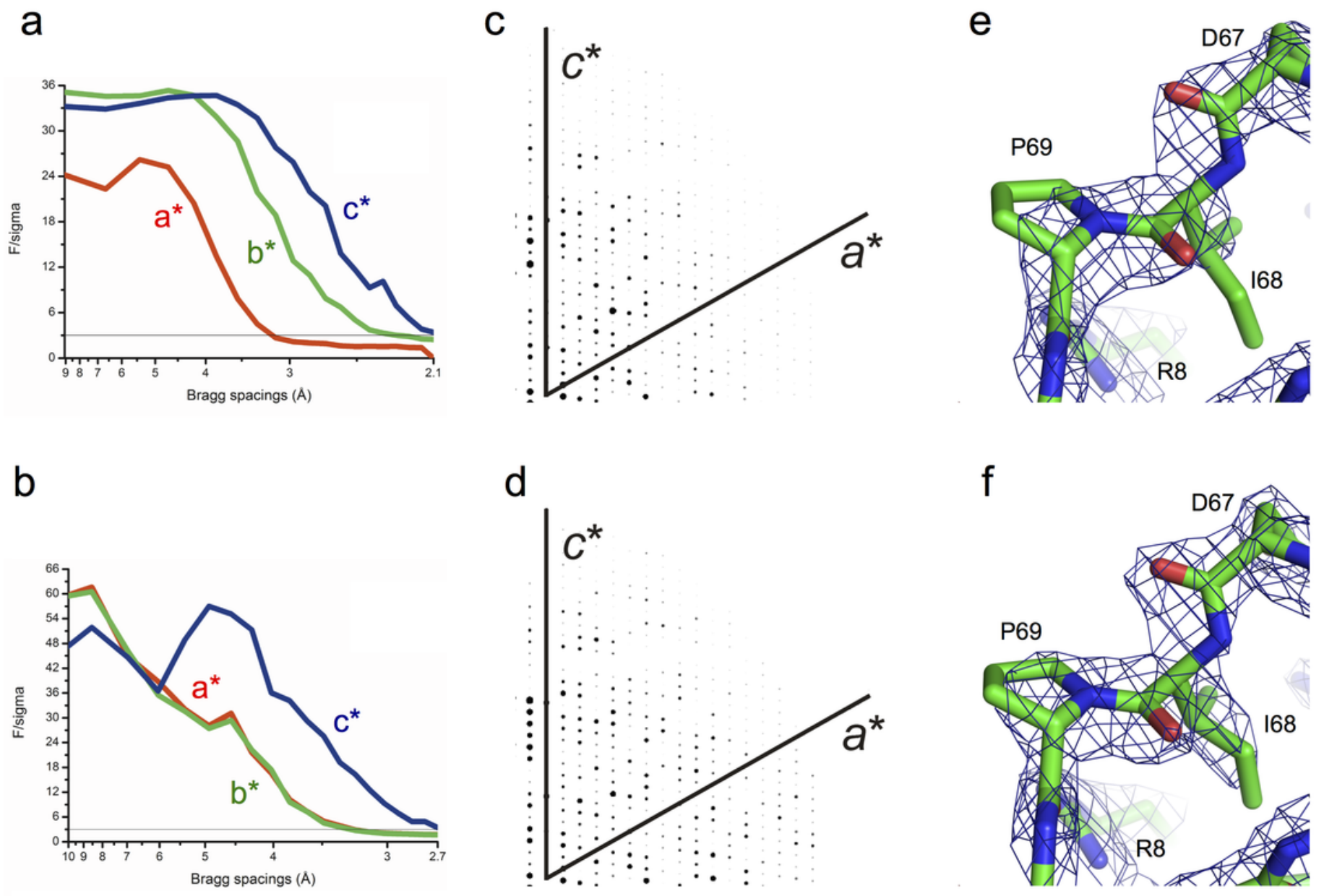


\section{3}

Figure 3. Structure and conserved packing interfaces of C-terminally extended NS6 proteases.

(a) Superposition of the four molecules in the asymmetric unit of crystals of NS6 1' (coloured various shades of blue). The $\beta$-strands are labelled, as are the $\mathrm{N}$ - and $\mathrm{C}$-termini and the conserved side-chains of catalytic triad. Note that the active site $\mathrm{C} 139$ has been replaced by A139 in all structures reported here. (b) A conserved packing arrangement observed for the mature NS6 protease (NS6 1-183) (Leen, Baeza \& Curry, 2012) and three of the structures solved in the present work (NS6 2', NS6 4' and NS6 4' 2|3). In this panel the label for the Nterminus is adjacent to the $\mathrm{N}$-terminal helix that is central to the packing interface. This packing arrangement is not conserved in the NS6 $1^{\prime}$ crystals but the B chain of NS6 $1^{\prime}$ is included, superposed on the molecule on the left-hand side, to further illustrate the variation in the C-termini of the different constructs. Key features are labelled to facilitate comparison of panels $a$ and $b$.
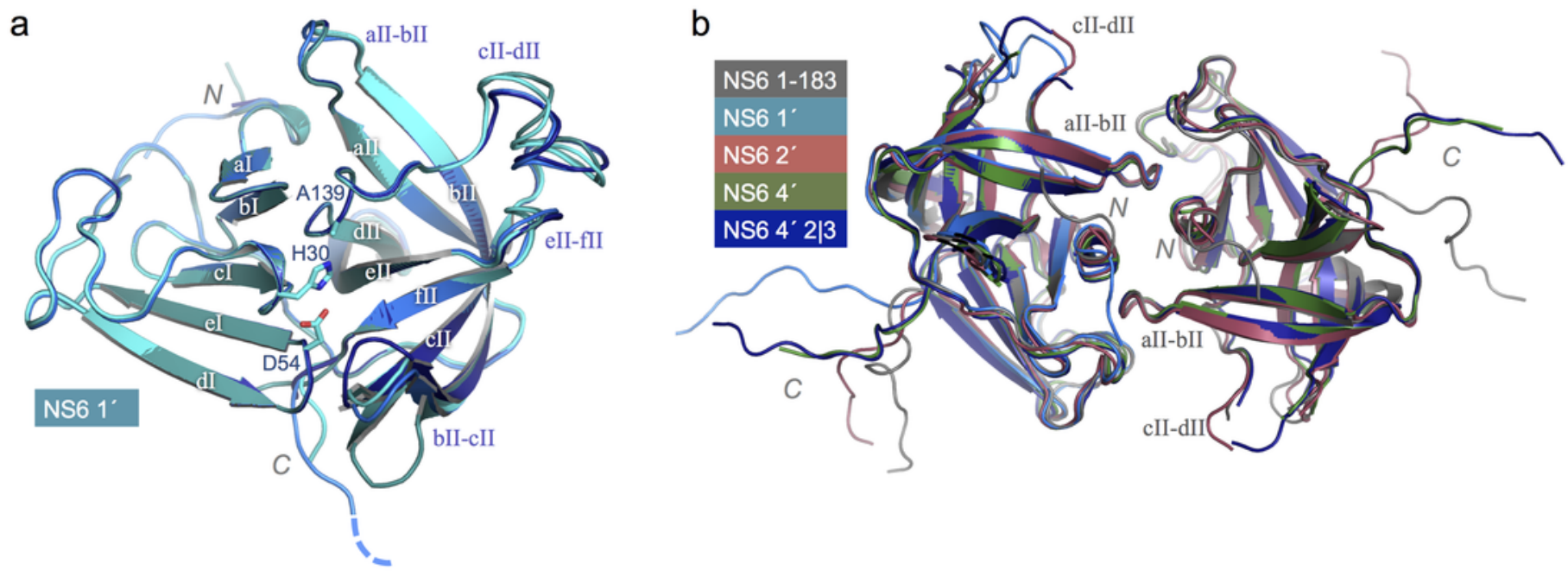


\section{4}

Figure 4. Comparison of the packing arrangements of C-terminally extended NS6 proteases.

(a) The mature NS6 protease (NS6 1-183) (Leen, Baeza \& Curry, 2012). Chains which interact via C-termini are coloured blue and orange. This colour-scheme is maintained throughout the figure; note also that the orientation of the blue chain is the same in each panel. In all panels the side-chains of the active site residues/mutations C139A, H30 and D54 are shown as sticks; their locations are indicated by dotted ovals. The presence of two-fold symmetry axes between interacting molecules are indicated by a solid black oval, although the views shown are only approximately along these axes. (b) NS6 1' -the interaction is between a pair of symmetry-related $B$ chains. An additional pair of symmetry-related $C$ chains -which also contact the extended C-terminus of the B chains - is shown in white (c) NS6 $2^{\prime}$. In this case the interaction is between the two chains in the asymmetric unit. (d) NS6 $4^{\prime}$-here there is only one chain in the asymmetric unit and the interaction is not symmetric. (e) NS6 4' $2 \mid 3$ -here again the interaction between chain $B$ (blue) of one asymmetric unit and chain $A$ (orange) of another is not symmetric. (f) NS6 4' $2 \mid 3$-a second but very similar mode of interaction in these crystals between chain A (blue) of one asymmetric unit and chain $B$ (orange) of another. 

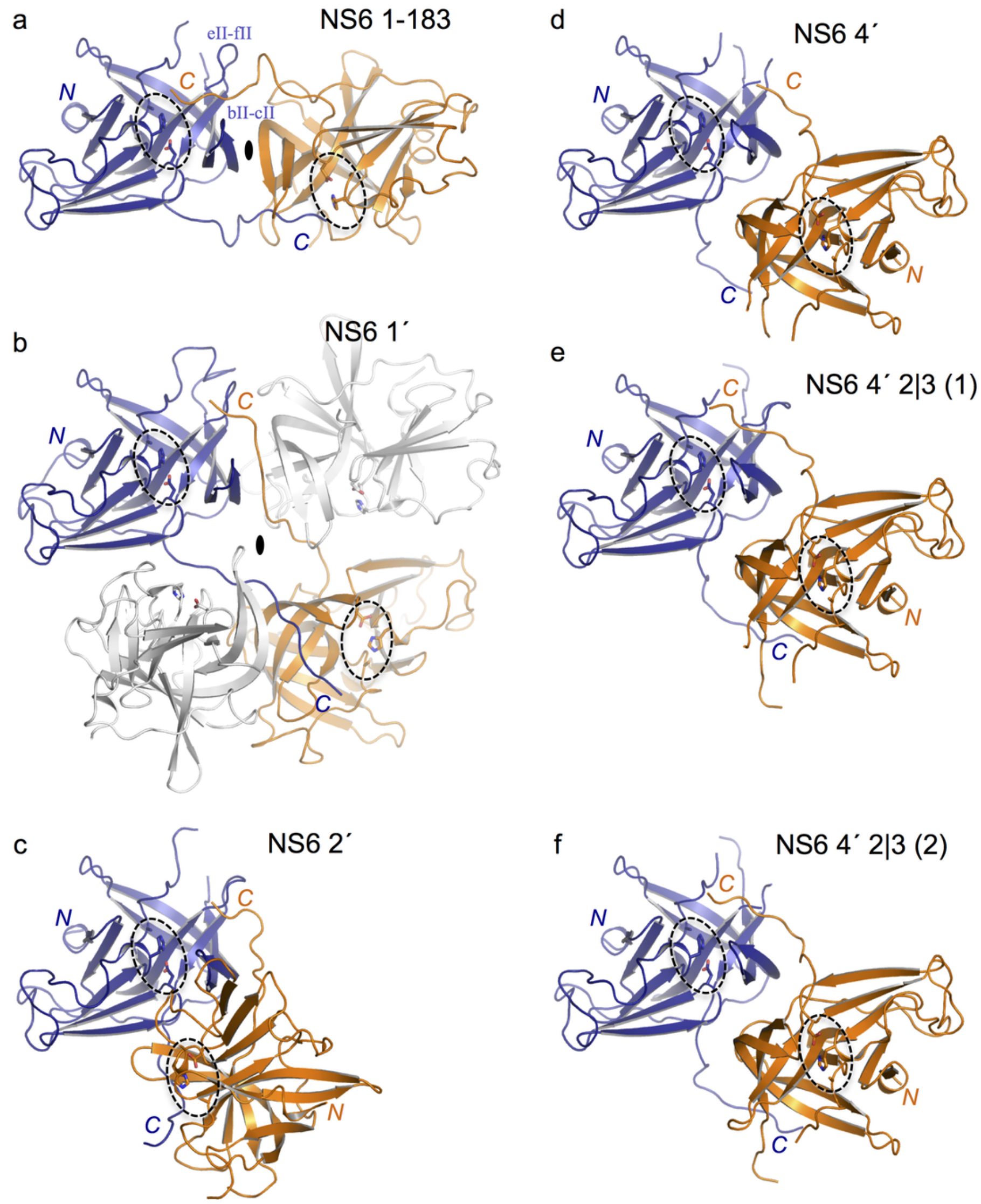